\title{
Socioeconomic Factors Impact Inpatient Mortality in Pediatric Lymphoma Patients
}

\author{
Yana Puckett ${ }^{1}$, Anh Ta ${ }^{2}$ \\ 1. Department of Surgery, Texas Tech University Health Sciences Center 2. School of Medicine, Saint \\ Louis University
}

$\square$ Corresponding author: Anh Ta, tanl@slu.edu

Disclosures can be found in Additional Information at the end of the article

\section{Abstract}

Purpose: Our objective was to determine the risk factors for inpatient mortality of pediatric patients diagnosed with lymphoma through the utilization of a large national pediatric database.

Methods: This cross-sectional study uses data from the Healthcare Cost and Utilization Project Kids' Inpatient Database (HCUP KID) for the year of 2012 to estimate the risk factors for inpatient mortality for pediatric patients diagnosed with lymphoma. All patients diagnosed with lymphoma between the ages of one and 18 years were included. Chi-square test was used to analyze categorical variables. Independent t-test was used to analyze continuous variables.

Results: A total of 2,908 study subjects with lymphoma were analyzed. Of those, $56.1 \%$ were male and the average age was three years old. Total inpatient mortality was $1.2 \%$ or 34 patients. We found that patients with four or more chronic conditions were much more likely to die while hospitalized ( $\mathrm{p}$ 0.0001). In addition, we also saw that patients with median household incomes below $\$ 47,999$ dollars ( $p=0.05)$ having a need for a major procedure $(p=0.008)$ were associated with inpatient mortality. Congestive heart failure, renal failure, coagulopathy, metastatic disease, and electrolyte abnormalities were all found to be associated with inpatient mortality.

Conclusions: Pediatric lymphoma mortality in children is not only influenced by their medical condition but also by their socioeconomic condition as well.

Categories: Pediatrics, Oncology

Keywords: pediatric cancer, lymphoma, socioeconomic status, chronic illness, pediatric malignancies

Received 04/22/2016 Review began 05/03/2016 Review ended 05/12/2016 Published 05/27/2016

\section{(๑) Copyright 2016}

Puckett et al. This is an open access article distributed under the terms of the Creative Commons Attribution License CC-BY 3.0., which permits unrestricted use, distribution, and reproduction in any medium, provided the original author and source are credited.

\section{Introduction}

Lymphoma is the third most frequent type of cancer in children, making up $11.5 \%$ of all childhood malignancies [1]. For children, ages 0-14, approximately 620 cases of non-Hodgkin's lymphoma (NHL) and 380 cases of Hodgkin's lymphoma (HL) are diagnosed annually. Over the past 40 years, significant improvements have been made in the diagnosis and treatment of lymphoma. The five-year survival rate of HL has increased from 87\% from 1975-1979 to 97\% from 2003-2009. Survival rates for NHL increased from $47 \%$ to $85 \%$ during the same time period $[2]$.

Poor survival from lymphoma is strongly associated with a variety of factors. In HL, pretreatment factors that contribute to poor overall survival (OS) include B symptoms, 
extranodal disease, bulky disease, hemoglobin less than $11.0 \mathrm{~g} / \mathrm{dL}$, nodular sclerosis histology, and erythrocyte sedimentation rate of greater than $50 \mathrm{~mm} / \mathrm{h}$ [3-5]. Poor response to initial therapy contributes to poor survival as well [6]. In NHL, factors that affect survival include response to therapy, stage and site of disease at diagnosis, and genetic abnormalities [7-10]. Central nervous system (CNS) and bone marrow (BM) involvement are strong predictors of outcome. A randomized study of high-risk NHL involving the CNS and BM reported a 30\% event-free survival (EFS) in patients who did not respond to initial treatments compared to $97 \%$ in those who did. The same study also reported that patients with BM involvement only and CNS involvement only had a four year EFS of $88 \%$ and $82 \%$, respectively. In patients with both CNS and BM involvement, the EFS drops to $61 \%$ [7].

The prognosis of lymphoma is also determined by factors that lead to inpatient mortality. Controlling infection is critical in lymphoma management because hematologic cancers are strongly associated with sepsis [11]. Major procedures, including central lines, positive pressure ventilation, and renal replacement therapy, are also linked to higher mortality [11-12]. Lastly, the treatment of lymphoma can cause tumor lysis syndrome, subsequent renal failure, and a significantly poorer prognosis.

Among these prognostic factors, however, the impact of various comorbidities that are present in children with lymphoma are largely unexplored, despite them being widely researched in adult cancers. While this is due to older patients presenting with more comorbidities, comorbidities are nevertheless strongly associated with lower survival and their presence in children needs to be further explored [13].

Furthermore, while there is extensive research on prognosis based on the properties and presentation of lymphoma, there is little research on how non-lymphoma-related factors can affect survival. The most commonly discussed factors are race, sex, and age. The Surveillance, Epidemiology, and End Results Program (SEER) report shows white children having a slightly higher survival than black children in HL and children younger than 10 having a better survival in NHL [14]. Although socioeconomic factors were associated with an increased risk of disease, the study did not report if they had an impact on survival. A separate study in Switzerland reported an association between paternal education status and lymphoma survival [15]. However, due to the differences in the Swiss economic and healthcare system, there is a need to explore the impact of socioeconomic factors in the United States (US).

\section{Materials And Methods}

Utilizing data from the Healthcare Cost and Utilization Project Kids' Inpatient Database (HCUP KID) compiled for the year 2012, we performed a retrospective analysis of all pediatric lymphoma patients in the United States. Patient-related variables obtained from the database included age (years), sex, race (Caucasian, African American, Hispanic, Asian or Pacific Islander, Native American, and other), in-hospital mortality, and comorbidities associated with each patient. Each record contained discharge diagnosis and procedure codes defined by the 15th International Classification of Diseases, Ninth Revision, Clinical Manifestations (ICD-9$\mathrm{CM})$.

Comorbid conditions were identified on the basis of both the ICD-9 diagnosis and procedure codes and the Clinical Classification Software diagnosis and procedure classifications. The Statistical Package for the Social Sciences (SPSS) was utilized for data analysis. Chi-square test was used to analyze categorical variables. Independent t-test was used to analyze continuous variables. Estimations with P values less than .05 were considered statistically significant.

Ethical approval was obtained from the Institutional Review Board (IRB) at Saint Louis 


\section{Cureus}

University prior to the start of the study. Informed consent was obtained at the time of initial treatment.

\section{Results}

A total of 2,908 study subjects with lymphoma were analyzed. Of those, $56.1 \%$ were male and the average age was three years. Caucasians comprised $50.9 \%$ of the population, followed by Hispanics (18.8\%), and African-Americans (12.5\%). The average length of stay was 6.16 days. The average patient had a total of approximately two procedures and three chronic conditions. The most common expected payer was private insurance with Medicaid being second. Total inpatient mortality was $1.2 \%$ or 34 patients (Table 1 ).

Demographics of Study Population $(n=2908)$

Gender (Male)

Mean Age (SD)

Mean LOS Days (SD)

Mean Number of Procedures (SD)

Mean Number of Chronic Conditions (SD)

Mortality

\section{Primary Expected Payer}

Medicare

Medicaid

Private Insurance

Self-Pay

No Charge

Race

White

Black

Hispanic

Asian or Pacific Islander

Native American

Other

\section{Region of Hospital}

Northeast

Midwest

South
$19.3 \%(561)$

$23.0 \%(670)$

$56.1 \%$

2.97 (1.1)

$6.16(10.0)$

1.7 (2.4)

3.2 (2.0)

$1.2 \%(34)$

$0.9 \%(27)$

$35.4 \%(1,028)$

$54.6 \%(1,587)$

$2.9 \%(84)$

$5.9 \%(173)$

$50.9 \%(1,481)$

$12.5 \%(364)$

$18.8 \%(546)$

$3.7 \%$ (108)

$0.3 \%(9)$

$4.7 \%$ (136)

$35.6 \%(1,036)$ 


\section{Cureus}

West

Median Household Income (USD)

$1-38,999$

$39,000-47,999$

$48,000-62,999$

$63,000+$

\section{Most Common Procedure Type}

Blood Transfusion

$19.2 \%(557)$

Enteral and Parenteral Nutrition

$3.5 \%(103)$

Cancer Chemotherapy

Other

Nontherapeutic Skin Procedures

Diagnostic Spinal Tap

Epidural/Spinal Catheter Insertion

Central Line Catheter Placement

Bone Marrow Transplant

Respiratory Intubation/Mechanical Ventilation

$1.1 \%(32)$

\section{TABLE 1: Demographics of Study Population $(n=2908)$}

SD: Standard Deviation; n: Population Number; LOS: Length of Stay; USD: United States Dollars

In performing a comparison of patient demographics in pediatric lymphoma patients in terms of inpatient mortality, patients with four or more chronic conditions were much more likely to die while hospitalized $(\mathrm{p}<0.0001)$. In addition, we also saw that patients with median household incomes below $\$ 47,999$ dollars were more predisposed to inpatient mortality compared to those making $\$ 48,000$ and above $(\mathrm{p}=0.05)$. The need for a major procedure was associated with inpatient mortality as well $(\mathrm{p}=0.008)$ (Table 2$)$. There were no statistically significant associations found between gender, race, primary expected payer, and region of the hospital.

Mortality \% (n=34)

$52.9 \%(18)$

Gender (Female)

Race

African-American
P-Value

$44.9 \%(1,258)$

0.185 


\section{Cureus}

\begin{tabular}{|c|c|c|c|}
\hline Caucasian & $23.5 \%(8)$ & $12.7 \%(356)$ & 0.208 \\
\hline Hispanic & $14.7 \%(5)$ & $19.3 \%(541)$ & \\
\hline Other & $17.6 \%(6)$ & $3.4 \%(106)$ & \\
\hline Primary Expected Payer & & & \\
\hline Medicare & $5.9 \%(2)$ & $0.01 \%(25)$ & \\
\hline Medicaid & $32.4 \%(11)$ & $36.3 \%(1,017)$ & \\
\hline Private Insurance & $50.0 \%(17)$ & $56.1 \%(1,570)$ & 0.045 \\
\hline Self-Pay & $2.9 \%(1)$ & $3.0 \%$ (83) & \\
\hline No Charge & $8.8 \%(3)$ & $6.1 \%(170)$ & \\
\hline Region of Hospital & & & \\
\hline Northeast & $17.7 \%(6)$ & (555) & \\
\hline Midwest & $23.5 \%(8)$ & (662) & \\
\hline South & $41.2 \%(14)$ & $(1,022)$ & \\
\hline West & $17.7 \%(6)$ & (635) & \\
\hline Number of Chronic Conditi & & & \\
\hline 1 & $5.9 \%(2)$ & $19.8 \%(441)$ & \\
\hline 2 & $0.0 \%(0)$ & $28.8 \%(808)$ & \\
\hline 3 & $11.8 \%(4)$ & $24.1 \%(675)$ & $<0.001$ \\
\hline 4 & $20.6 \%(7)$ & $15.0 \%(421)$ & \\
\hline $5+$ & $61.8 \%(21)$ & $18.9 \%(529)$ & \\
\hline Median Household Income & & & \\
\hline $1-38,999$ & $35.3 \%(12)$ & $25.8 \%(723)$ & \\
\hline $39,000-47,999$ & $38.2 \%(13)$ & $24.2 \%(677)$ & \\
\hline $48,000-62,999$ & $11.8 \%(4)$ & $23.4 \%(655)$ & \\
\hline $63,000+$ & $14.7 \%(5)$ & $27.1 \%(759)$ & \\
\hline Need for Major Procedure & $76.5 \%(26)$ & $45.3 \%(1268)$ & 0.008 \\
\hline
\end{tabular}

TABLE 2: Comparison of Patient Demographics in Pediatric Lymphoma Patients in the United States with In-Hospital Mortality Being the Outcome, 2012 ( $n=2,908)$

Chi-Square: Categorical Variables

Independent Samples T-Test: Continuous Variables

n: Population Number 
A comparison of comorbidities in pediatric lymphoma patients was performed as well. A total of 25 comorbidities were analyzed. Of those, congestive heart failure $(p=0.001)$, coagulopathy $(p<0.0001)$, fluid and electrolyte disorders $(p<0.0001)$, metastatic cancer $(p=0.024)$, neurological disorder $(p=0.027)$, and renal failure $(p=0.006)$ were found to be statistically significant in terms of being a risk factor for inpatient mortality (Table 3).

\begin{tabular}{|c|c|c|c|}
\hline & Mortality \% (n=34) & $\begin{array}{l}\text { No Mortality \% } \\
(\mathrm{n}=2,801)\end{array}$ & P-Value \\
\hline Obesity & $0.0 \%(0)$ & $2.6 \%(73)$ & N/A \\
\hline Deficiency Anemias & $14 \%(5)$ & $9.1 \%(254)$ & 0.180 \\
\hline Rheumatoid Arthritis/Collagen Vascular Disease & $0.0 \%(0)$ & $0.4 \%(11)$ & N/A \\
\hline Chronic Blood Loss Anemia & $0.0 \%(0)$ & $0.5 \%(14)$ & N/A \\
\hline Congestive Heart Failure & $8.8 \%(3)$ & $0.4 \%(12)$ & 0.001 \\
\hline Chronic Pulmonary Disease & $2.9 \%(1)$ & $8.2 \%(229)$ & 0.236 \\
\hline Coagulopathy & $41 \%$ (14) & $11 \%(314)$ & $<0.001$ \\
\hline Depression & $5.9 \%(2)$ & $4.8 \%(135)$ & 0.481 \\
\hline Diabetes, Uncomplicated & $2.9 \%(1)$ & $0.9 \%(26)$ & 0.273 \\
\hline Diabetes, with chronic complications & $0.0 \%(0)$ & $0.1 \%(2)$ & N/A \\
\hline Drug Abuse & $2.9 \%(1)$ & $1.7 \%(48)$ & 0.441 \\
\hline $\begin{array}{l}\text { Hypertension (combined uncomplicated and } \\
\text { complicated) }\end{array}$ & $12 \%(4)$ & $6.2 \%(174)$ & 0.151 \\
\hline Hypothyroidism & $0.0 \%(0)$ & $2.1 \%(59)$ & N/A \\
\hline Liver Disease & $5.9 \%(2)$ & $1.4 \%(39)$ & .082 \\
\hline Fluid and Electrolyte Disorders & $76 \%(26)$ & $19 \%(540)$ & $<0.001$ \\
\hline Metastatic Cancer & $5.9 \%(2)$ & $0.7 \%(19)$ & 0.024 \\
\hline Neurological Disorder & $12 \%(4)$ & $3.4 \%(95)$ & 0.027 \\
\hline Paralysis & $5.9 \%(2)$ & $1.2 \%(35)$ & 0.069 \\
\hline Peripheral Vascular Disorders & $2.9 \%(1)$ & $0.2 \%(5)$ & 0.068 \\
\hline Psychosis & $0.0 \%(0)$ & $1.3 \%(37)$ & N/A \\
\hline Pulmonary Circulation Disorders & $2.9 \%(1)$ & $0.7 \%(19)$ & 0.210 \\
\hline Renal Failure & $12 \%(4)$ & $2.1 \%(59)$ & 0.006 \\
\hline Solid Tumor without Metastasis & $0.0 \%(0)$ & $0.6 \%(18)$ & N/A \\
\hline
\end{tabular}




\section{Cureus}

Valvular Disease

$2.9 \%(1)$

$0.4 \%(11)$

Weight Loss

$8.8 \%(3)$

$0.4 \%(11)$

0.160

TABLE 3: Comparison of Comorbidities in Pediatric Lymphoma Patients in the United States with In-Hospital Mortality Being the Outcome, 2012 ( $n=2,908)$

Chi-Square: Categorical Variables

Independent Samples T-Test: Continuous Variables

N/A: Not Applicable

n: Population Number

\section{Discussion}

\section{Main findings of the study}

In this study, household income, the need for a major procedure, the number of chronic conditions, and the number of comorbidities were identified as important risk factors for inpatient hospital mortality in pediatric lymphoma patients.

Children in families with a median income of less than $\$ 47,999$ had a 1.4 times higher mortality risk while hospitalized than children in families of higher income. This income is lower than the median income in the US as reported by the US Census Bureau [16]. This is consistent with previous analysis between lower socioeconomic status and HL survival and also with socioeconomic studies in adults with diffuse large B-cell lymphoma [17-18].

However, we also found that there is no significany difference in inpatient mortality among different expected primary payers. This result is different from a previous study that suggests uninsured children have a higher inpatient mortality overall. The same study suggests that poorer outcomes may be due to uninsured families depending on emergency services as their source of care and children having more advanced stages when they present [19]. Our data does not indicate that type of insurance affects inpatient mortality in pediatric lymphoma patients. There was no statistically significant difference in mortality between lymphoma patients on medicaid, medicare, private insurance, or self-pay status. Future research needs to be done to see if patients with lower socioeconomic status have more advanced disease when they present. Other socioeconomic factors facing families with lower incomes may be involved. For example, in a high-income country like Switzerland, where there is an above-average educational system and universal health care, lower education status of the father is still associated with a lower lymphoma survival [15]. Therefore, other socioeconomic factors need to be assessed and considered.

To the best of our knowledge, there are no previous studies investigating causes of inpatient mortality in pediatric lymphoma patients. Several studies report the most common cause of death in lymphoma to be infection, renal failure, and hemorrhage [11-12, 20-22]. Our findings echo these studies as renal failure and coagulation abnormalities were significantly associated with inpatient mortality in pediatric lymphoma patients.

Hematologic cancers are more likely to be associated with sepsis and viral infection, which is associated with a higher mortality. This problem is further worsened by both malignancy and 
treatments causing immunosuppression [11]. Early mortality in advanced mature B-cell malignancies has been observed with extensive surgical procedures, although the result was not statistically significant [22]. Our study found that patients who had the need for a major procedure were much more likely to have an inpatient mortality. However, the top three procedures cited as "major" in the database for those with inpatient mortality were mechanical intubation (32.4\%), blood transfusion (14.7\%), and enteral or parenteral nutrition (11.8\%). These results can generally be explained by these procedures being used only for patients who are already in worse conditions.

In addition, pediatric cancer patients have a higher risk of hospital-associated infections compared to other pediatric patients, especially with central lines [12]. The need for prolonged parenteral nutrition further predisposes these pediatric cancer patients to central line infections [23]. Hematologic cancer patients are also more likely to receive noninvasive positive pressure ventilation and renal replacement therapy in the PICU compared to other cancers and equally likely to use invasive positive pressure ventilation. Each intervention is associated with higher mortality in the PICU [11-12]. Lymphoma patients also commonly need blood transfusions, which can have immunosuppressive effects, putting the patient at risk of infection or viral reactivation [24]. The need for a procedure needs to be carefully assessed, especially in immunocompromised lymphoma patients, so that children are not put at risk of infection and inpatient death.

Having more than four chronic illnesses is a significant risk factor for inpatient mortality, consistent with past studies in adults [13]. Pediatric cancer patients who present with organ involvement and failure had a higher frequency of death [12]. Specific comorbidities associated with increased inpatient mortality were congestive heart failure, coagulopathy, fluid and electrolyte disorder, metastatic disorder, neurological disorder, and renal failure. Higher Pediatric Risk of Mortality 3 (PRISM3) score based on cardiovascular, neurologic, acid-base chemistry, and hematologic derangements were associated with a higher risk of PICU mortality [11]. Metastatic disorder, extranodal disease, and bulky disease are also associated with a worse prognosis in lymphoma, a result also observed in our study [3]. Coagulopathy and neurological disorders being risk factors are supported by BM and CNS involvement in lymphoma, leading to lower survival independently and an even lower survival when patients have involvement of both systems [7]. This suggests inpatient mortality and long-term survival can be improved by effective screening to catch disease before extranodal and metastatic spread, especially to the CNS and BM.

Fluid and electrolyte disorders and renal failure are a common result of tumor lysis syndrome, a frequent complication and risk of mortality in lymphoma [25]. Tumor lysis syndrome typically occurs after the initiation of chemo or radiotherapy and causes hyperphosphatemia, hypocalcemia, hyperkalemia, hyperuricemia, and ultimately acute renal failure [26]. With renal failure being one of the most common causes of death in lymphoma patients, careful monitoring, prevention, and treatment of renal dysfunction is essential in preventing inpatient mortality in lymphoma [20, 27].

\section{Limitations of the study}

This study has a number of limitations. We are unable to collect other socioeconomic factors that may affect the outcome. As shown by previous studies, other factors, such as educational status and compliance, may affect survival [15, 18]. However, if we expanded our data collection to include more variables, there are factors that may not be easily collected in a patient's chart retrospectively. This can include the parents' occupation, community support, and other factors that contribute to low socioeconomic status.

We are also limited by the lymphoma-related data provided by the Healthcare Cost and 
Utilization ProjectKids' Inpatient Database. From this database, we are unable to determine the different types of lymphoma and if the comorbidities are independent of the lymphoma or are results of the lymphoma. We also do not know from the database if the comorbidities were also diagnosed at the time of lymphoma diagnosis or if they were chronic conditions the child was already known to have. Therefore, we cannot know if the increase in mortality is due to the lymphoma disrupting the organ system or due to the existing dysfunction of that system exacerbating the lymphoma. This relationship needs to be further explored to determine how to monitor and manage comorbidities and improve outcomes. Furthermore, our database does not have long-term outcomes and survival, and we are unable to determine if the factors that contribute to inpatient mortality have an effect on long-term survival. In addition, factors, such as expected primary payer and comorbidities that are not identified as risk factors for inpatient mortality, may have an impact on long-term survival. Since many past studies of mortality have been on pediatric cancers in general rather than lymphoma, more studies need to be done to see if factors that affect pediatric cancer mortality affect lymphomas more specifically.

Lastly, this is a retrospective study, and thus, we are unable to determine how the comorbidities were managed and whether more effective management of the specific risk factors led to better survival. This relationship can be explored in future studies to develop better plans to manage pediatric lymphoma patients with comorbidities.

\section{Conclusions}

In this national children's database study, we found that pediatric lymphoma mortality in children is not only influenced by their medical condition but also by their socioeconomic condition as well. Medical conditions that contribute to inpatient mortality include comorbidities, the need for a major procedure, and metastatic and extranodal disease. Patients with low median family income are found to have higher mortality. One possible explanation for this result is that patients with low family income may have both higher number comorbidities and a more progressed disease at diagnosis, leading to a higher rate of mortality. We also found no significant difference in mortality among different expected primary payers, indicating that it is not enough for families to be insured. Other factors may exist including parent education level and access to care that contribute to a delay in diagnosis. Future research needs to be done to see if patients with a lower socioeconomic status present with more advanced disease and what factors contribute to a delay in diagnosis.

\section{Additional Information \\ Disclosures}

Human subjects: Consent was obtained by all participants in this study. Saint Louis University Institutional Review Board issued approval N/A. Because we used a national database with no identifiers present in it, the IRB at Saint Louis University determined that we were exempt from submitting a formal IRB for this study. Similarly, because this is a retrospective study with no identifiers, obtaining patient consent is not applicable. Animal subjects: All authors have confirmed that this study did not involve animal subjects or tissue. Conflicts of interest: In compliance with the ICMJE uniform disclosure form, all authors declare the following:

Payment/services info: All authors have declared that no financial support was received from any organization for the submitted work. Financial relationships: All authors have declared that they have no financial relationships at present or within the previous three years with any organizations that might have an interest in the submitted work. Other relationships: All authors have declared that there are no other relationships or activities that could appear to have influenced the submitted work.

\section{References}


1. Kaatsch P: Epidemiology of childhood cancer. Cancer Treat Rev . 2010, 36:277-85. 10.1016/j.ctrv.2010.02.003

2. Ward E, DeSantis C, Robbins A, Kohler B, Jemal A: Childhood and adolescent cancer statistics, 2014. CA Cancer J Clin. 2014, 64:83-103. 10.3322/caac.21219

3. Smith RS, Chen Q, Hudson MM, Link MP, Kun L, Weinstein H, Billett A, Marcus KJ, Tarbell NJ, Donaldson SS: Prognostic factors for children with Hodgkin's disease treated with combinedmodality therapy. J Clin Oncol. 2003, 21:2026-33. 10.1200/JCO.2003.07.124

4. Rühl U, Albrecht M, Dieckmann K, Lüders H, Marciniak H, Schellenberg D, Wickmann L, Dörffel W: Response-adapted radiotherapy in the treatment of pediatric Hodgkin's disease: an interim report at 5 years of the German GPOH-HD 95 trial. Int J Radiat Oncol Biol Phys. 2001, 51:1209-18. 10.1016/S0360-3016(01)01798-9

5. Vecchi V, Pileri S, Burnelli R, Bontempi N, Comelli A, Testi AM, Carli M, Sotti G, Rosati D, Di Tullio MT, Grazia G, Massolo F, Aricó M, Colella R, Pession A, Rondelli R, Paolucci G:

Treatment of pediatric hodgkin disease tailored to stage, mediastinal mass, and age an italian (aieop) multicenter study on 215 patients. Cancer. 1993, 72:2049-57. 10.1002/10970142(19930915)72:6<2049::AID-CNCR2820720642>3.0.CO;2-V

6. Landman-Parker J, Pacquement H, Leblanc T, Habrand JL, Terrier-Lacombe MJ, Bertrand Y, Perel Y, Robert A, Coze C, Thuret I, Donadieu J, Schaison G, Leverger G, Lemerle J, Oberlin O: Localized childhood Hodgkin's disease: response-adapted chemotherapy with etoposide, bleomycin, vinblastine, and prednisone before low-dose radiation therapy-results of the French Society of Pediatric Oncology Study MDH90. J Clin Oncol. 2000, 18:1500-1507.

7. Cairo MS, Gerrard M, Sposto R, Auperin A, Pinkerton CR, Michon J, Weston C, Perkins SL, Raphael M, McCarthy K, Patte C; FAB LMB96 International Study Committee: Results of a randomized international study of high-risk central nervous system B non-Hodgkin lymphoma and B acute lymphoblastic leukemia in children and adolescents. Blood. 2007, 109:2736-43. 10.1182/blood-2006-07-036665

8. Salzburg J, Burkhardt B, Zimmermann M, Wachowski O, Woessmann W, Oschlies I, Klapper W, Wacker HH, Ludwig WD, Niggli F, Mann G, Gadner H, Riehm H, Schrappe M, Reiter A: Prevalence, clinical pattern, and outcome of CNS involvement in childhood and adolescent non-Hodgkin's lymphoma differ by non-Hodgkin's lymphoma subtype: a Berlin-FrankfurtMunster Group Report. J Clin Oncol. 2007, 25:3915-22. 10.1200/JCO.2007.11.0700

9. Poirel HA, Cairo MS, Heerema NA, Swansbury J, Aupérin A, Launay E, Sanger WG, Talley P, Perkins SL, Raphaël M, McCarthy K, Sposto R, Gerrard M, Bernheim A, Patte C; FAB/LMB 96 International Study Committee: Specific cytogenetic abnormalities are associated with a significantly inferior outcome in children and adolescents with mature B-cell non-Hodgkin's lymphoma: results of the FAB/LMB 96 international study. Leukemia. 2009, 23:323-31. 10.1038/leu.2008.312

10. Bonn BR, Rohde M, Zimmermann M, Krieger D, Oschlies I, Niggli F, Wrobel G, Attarbaschi A, Escherich G, Klapper W, Reiter A, Burkhardt B: Incidence and prognostic relevance of genetic variations in T-cell lymphoblastic lymphoma in childhood and adolescence. Blood. 2013, 121:3153-60. 10.1182/blood-2012-12-474148

11. Zinter MS, DuBois SG, Spicer A, Matthay K, Sapru A: Pediatric cancer type predicts infection rate, need for critical care intervention, and mortality in the pediatric intensive care unit. Intensive Care Med. 2014, 40:1536-44. 10.1007/s00134-014-3389-2

12. Faraci M, Bagnasco F, Giardino S, Conte M, Micalizzi C, Castagnola E, Lampugnani E, Moscatelli A, Franceschi A, Carcillo JA, Haupt R: Intensive care unit admission in children with malignant or nonmalignant disease: incidence, outcome, and prognostic factors: a single-center experience. J Pediatr Hematol Oncol. 2014, 36:e403-9. 10.1097/MPH.0000000000000048

13. Søgaard M, Thomsen RW, Bossen KS, Sørensen HT, Nørgaard M: The impact of comorbidity on cancer survival: a review. Clin Epidemiol. 2013, 5:3-29. 10.2147/CLEP.S47150

14. Percy CL, Smith MA, Linet M, Gloeckler Ries LA, Friedman DL: Lymphomas and reticuloendothelial neoplasms. Cancer Incidence and Survival among Children and Adolescents: United States SEER Program 1975-1995. 1999, NIH Pub. No. 99-4649:35-49. Accessed: April 21, 2016: http://seer.cancer.gov/archive/publications/childhood/childhoodmonograph.pdf.

15. Adam M, Rueegg CS, Schmidlin K, Spoerri A, Niggli F, Grotzer M, von der Weid NX, Egger M, Probst-Hensch N, Zwahlen M, Kuehni CE; Swiss Paediatric Oncology Group (SPOG) and the 
Swiss National Cohort Study (SNC): Socioeconomic disparities in childhood cancer survival in Switzerland. Int J Cancer. 2016, 138:2856-66. 10.1002/ijc.30029

16. US Bureau of the Census, Real Median Household Income in the United States . (2014). Accessed: April 6, 2016: https://research.stlouisfed.org/fred2/series/MEHOINUSA672N.

17. Gupta S, Wilejto M, Pole JD, Guttmann A, Sung L: Low socioeconomic status is associated with worse survival in children with cancer: a systematic review. PLoS One. 2014, 9:e89482.

Accessed: April 6, 2016: http://journals.plos.org/plosone/article?

id=10.1371/journal.pone.0089482. 10.1371/journal.pone.0089482

18. Tao L, Foran JM, Clarke CA, Gomez SL, Keegan TH: Socioeconomic disparities in mortality after diffuse large B-cell lymphoma in the modern treatment era. Blood. 2014, 123:3553-62. 10.1182/blood-2013-07-517110

19. Abdullah F, Zhang Y, Lardaro T, Black M, Colombani PM, Chrouser K, Pronovost PJ, Chang DC: Analysis of 23 million US hospitalizations: uninsured children have higher all-cause inhospital mortality. J Public Health (Oxf). 2010, 32:236-44. 10.1093/pubmed/fdp099

20. Robertson CM, Stiller CA, Kingston JE: Causes of death in children diagnosed with nonHodgkin's lymphoma between 1974 and 1985. Arch Dis Child. 1992, 67:1378-83. 10.1136/adc.67.11.1378

21. Prakash G, Bakhshi S, Raina V, Bhatnagar S, Sharma A, Kumar L, Shukla NK, Julka PK, Rath GK: Characteristics and pattern of mortality in cancer patients at a tertiary care oncology center: report of 259 cases. Asian Pac J Cancer Prev. 2010, 11:1755-59.

22. Cervio C, Barsotti D, Ibanez J, Paganini H, Sara Felice M, Chantada GL: Early mortality in children with advanced mature B-cell malignancies in a middle-income country. J Pediatr Hematol Oncol. 2012, 34:e266-70. 10.1097/MPH.0b013e31826226b1

23. Wylie MC, Graham DA, Potter-Bynoe G, Kleinman ME, Randolph AG, Costello JM, Sandora TJ: Risk factors for central line-associated bloodstream infection in pediatric intensive care units . Infect Control Hosp Epidemiol. 2010, 31:1049-56. 10.1086/656246

24. Chow EJ, Holly EA: Blood transfusions and non-Hodgkin's lymphoma. Epidemiol Rev. 2002, 24:269-79. 10.1093/epirev/mxf005

25. Pastore G, Viscomi S, Mosso ML, Maule MM, Terracini B, Magnani C, Merletti F: Early deaths from childhood cancer. A report from the Childhood Cancer Registry of Piedmont, Italy, 19671998. Eur J Pediatr. 2004, 163:313-19. 10.1007/s00431-004-1425-x

26. Alavi S, Arzanian MT, Abbasian MR, Ashena Z: Tumor lysis syndrome in children with nonHodgkin lymphoma. Pediatr Hematol Oncol. 2006, 23:65-70. 10.1080/08880010500313561

27. Büyükpamukçu M, Varan A, Aydin B, Kale G, Akata D, Yalçin B, Akyüz C, Kutluk T: Renal involvement of non-Hodgkin's lymphoma and its prognostic effect in childhood. Nephron Clin Pract. 2005, 100:c86-91. 10.1159/000085053 